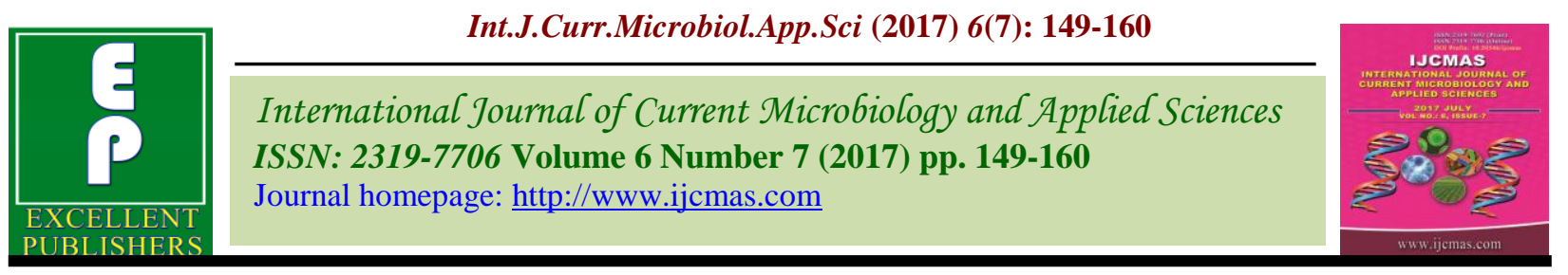

Original Research Article

https://doi.org/10.20546/ijcmas.2017.607.018

\title{
Differential Response of Root Morphology of Rice (Oryza sativa L.) Genotypes under Different Phosphorus Conditions
}

\author{
Datta P. Kakade*, Jyoti Singh, Mayur R. Wallalwar, Arun Janjal, \\ Anjali Gupta, Rishiraj Raghuvanshi, Miranda Kongbrailatpam, \\ Satish B. Verulkar and Shubha Banerjee \\ Department of Plant Molecular Biology and Biotechnology, College of Agriculture, \\ Indira Gandhi Krishi Vishwavidyalaya, Raipur, Chhattisgarh, Pin- 492012, India \\ *Corresponding author
}

A B S T R A C T

\begin{tabular}{|l|}
\hline Ke y w o r d s \\
Rice, \\
Phosphorus, \\
Root length, \\
Root surface area, \\
Root volume. \\
\hline Article Info \\
\hline $\begin{array}{l}\text { Accepted: } \\
\text { 04 June } 2017 \\
\text { Available Online: } \\
\text { 10 July } 2017\end{array}$ \\
\hline
\end{tabular}

Phosphorus (P) deficiency is a major constraint to rice production worldwide. Especially developing countries like India, having a limited access to $\mathrm{P}$ fertilizer. Genetic variations in rice in terms of root length, density of root hairs, modified root architecture, have been observed in low $\mathrm{P}$ concentration. Adaptive root modifications enhance $\mathrm{P}$ acquisition but are often associated with yield penalty. Rice genotypes with desired root traits along with better $\mathrm{P}$ utilization efficiency are required. The present study was conducted to evaluate the effect of phosphorus deficiency stress on root traits of diverse rice genotypes. Roots of rice genotypes grown in root rhizotron having soil $\mathrm{P}\left(\mathrm{P}_{2} \mathrm{O}_{5} \leq 7.59 \mathrm{~kg} / \mathrm{ha}\right)$, with and without $\mathrm{P}$ supplementation were compared for difference in total root length, lateral branching, surface area and volume. Significant difference was observed among the genotypes, due to inherent genetic makeup and also in their response to P deficiency stress. While an average decrease in all four root traits was recorded, few genotypes showed P deficiency induce lateral root development manifested as total root length, root surface area and volume. The three genotypes (Buddha, R-RF-78 and Cross 116) were identified as P deficiency tolerant rice genotypes, for use in further $\mathrm{P}$ efficient development of rice varieties.

\section{Introduction}

Phosphorus $(\mathrm{P})$ is the second most limiting macronutrient to plant growth and development after nitrogen. Either the soils are inherently deficient in $\mathrm{P}$ content or the soil $\mathrm{P}$ is not phyto-available due to immobilization of phosphate in soil, particularly acidic soil (Cordell et al., 2009; Wissuwa et al., 2005). Lack of plant available $\mathrm{P}$ constrains plant growth and reduced overall yield of rice (Cordell et al., 2009). P is the component of biomolecules like nucleic acid, phospholipids, etc. and is involved in major metabolic processes, of cells (Poirier et al., 2002). P deficiency affects overall plant growth and it has been assessed that 5.7 billion hectares of land worldwide are deficient in $\mathrm{P}$ (Batjes, 1997). Problem of $P$ deficiency is usually overcome by the application of phosphatic fertilizers (Abel et al., 2002). However, application of large amount of phosphatic fertilizer lead to higher $\mathrm{P}$ fixation and immobilization in soil rendering it less available to plant owing to changes in soil texture and $\mathrm{pH}$ due to long term application of 
fertilizers. Excessive use of $\mathrm{P}$ fertilizer also causes the soil and water pollution and increases economic burden over farmers. Limited and non-renewable rock phosphate reserves will escalate problem in the future (Aluwihare et al., 2016).

Few plant and microbial species have the ability to solubilize phosphate (Pi) bound to the soil particles by using different mechanisms. Mechanisms like exudation of organic acids and phosphatases leading to acidification of rhizosphere or a symbiotic mycorrhiza association have in making $\mathrm{P}$ more phyto-available (Jones, 1998; Richardson et al., 2009). Adaptive modifications in root traits in response to $\mathrm{P}$ deficiency stress have been observed for increased uptake of nutrient as well as water. As a key organ of rice plant; root performs vital functions like, acquisition of resources and anchorage of rice plant (Fitter, 2002; Rose et al., 2012; Wu et al., 2014). Previous studies showed that, increased root traits in rice were considerably positively associated with the uptake of macro- and micronutrients (Nielsen, 1979, Wang et al., 2016).

Under P deficient conditions, root of some rice genotypes undergoes morphological, anatomical and physiological changes to enhance the effective surface area for nutrient acquisition. Enhanced root traits lead to novel, more stress-tolerant crops and increased yield by increasing the capacity of the plant for soil exploration and, thus, results in improved water and nutrient acquisition (Wissuwa 2005; Paez-Garcia et al., 2016). Understanding of root morphology and physiology in response to $\mathrm{P}$ deficiency stress is important for evaluating the $\mathrm{P}$ deficiency tolerance capacity of rice genotypes.

Most common adaptions reported in root traits are increase in length, number of root hairs and lateral branching to exploit the soil space and enhance root-soil contact to increase $\mathrm{P}$ uptake (Kirk et al., 1996; Panigrahy, 2009; Sarker et al., 2009; Lynch, 2011). Since, root traits have been claimed to be critical for increasing yield under nutrient deficiency and water stresses (Lim et al., 2003; Lynch, 2007), these adaptive potentials of genotypes may be further utilized for effective translation of genetic potential into improved crop cultivars.

The present study was undertaken to screen and select the $\mathrm{P}$ tolerant genotypes on the basis of differential root growth response in contrasting conditions of $\mathrm{P}$ supplementation. A set of 46 diverse rice genotypes (Table 1) were selected for the study on the basis of their yield and genotyping for Pupl QTL specific marker (Chin et al., 2010) under rainfed condition (Gupta, 2016). The 46 rice genotypes were grown in rhizotron, since rhizotron culture facilitates measurements on root traits, as they minimize damage to root and root hairs during harvest and allow a precise control (Paez-Garcia et al., 2015).

\section{Materials and Methods}

The experiment was conducted at Department of Plant Molecular Biology and Biotechnology and research and instructional farm of Indira Gandhi Krishi Vishwavidyalaya, Raipur, (C.G.) during wet season-2015.

For phenotyping of root traits, a set of 46 diverse rice genotypes (Table 1) were grown in two nutrient conditions with 3 replications for 60 days in rhizotrons. The root rhizotron of size $(50 \times 50 \times 0.4 \mathrm{~cm})$ filled with $\mathrm{P}$ deficient soil $\left(\mathrm{P}_{2} \mathrm{O}_{5}<7.59 \mathrm{~kg} / \mathrm{ha}\right)$, made of transparent glass sheets.

The nutrient sufficient and deficient conditions were maintained with supply of nutrient with and without phosphorus as per 
the treatment were supplied on alternate days. The rhizotrons were placed at an angle of $35^{\circ}$ and number of leaves/plant, was recorded 60 days after sowing (DAS).

To record root traits rhizotron plates were split opened after 60 DAS and plants were harvested. Root systems were separated from the shoot and cleaned in running water. Finer soil particles still attached to the root were removed using a small painting brush and the roots were stored in $25 \% \mathrm{v} / \mathrm{v}$ ethanol, immediately after rinsing. Root systems were analysed using the flat-bed scanner by placing a transparent acrylic container having the cleaned root samples dispersed in water over the surface of the scanner (Epson Perfection V700 Photo) and scanned. To evaluate total root length, root surface area and root volume the images were analysed using WinRhizo software (Figure 1). The final outputs of the scanning obtained in form of an image and Microsoft excel data files for root parameters such as length, surface area and volume.

\section{Results and Discussion}

Root traits and number of leaves of rice genotypes were compared under contrasting conditions of $\mathrm{P}$ in rhizotron. On the basis of growth response of root traits and number of leaves genotypes are screened for $\mathrm{P}$ deficiency and categorized in the 3 groups likely tolerant, moderately tolerant and sensitive to P. Genotypes which having significantly decreased root traits in the $\mathrm{P}$ stressed condition as compared to controlled were groped in $\mathrm{P}$ sensitive genotypes (Figure 2). Genotypes which are not or least affected by the $\mathrm{P}$ treatment/stress are considered as moderately tolerant. Genotypes in which root traits are induced by the $\mathrm{P}$ stress were groped in $\mathrm{P}$ tolerant group (Figure 2), because in $\mathrm{P}$ deficiency root traits generally increase in tolerant genotypes (Lynch, 1995).
Significant variations were observed among all the genotypes, $\mathrm{P}$ treatments and interaction among them (Table 3). Overall analysis across the contrasting $P$ levels showed significant increase in all the root and shoot traits in controlled condition compared to $\mathrm{P}$ stressed condition (Table 2 and Figure 3 A, B, C, D and E). In tolerant genotypes, both of the root and shoot traits increased significantly in $\mathrm{P}$ deficient condition showing their higher efficiency for P uptake. Most of the genotypes having good growth in the controlled condition but they fail to perform in the $\mathrm{P}$ stressed condition.

\section{Root and shoot traits}

\section{Root Length (RL)}

Root length is the length from the base of the root to the end of the root tip it may include the length of the single long root. In P, deficient condition overall RL $(1862.7 \mathrm{~cm})$ was decreased compared to overall RL of sufficient $\mathrm{P}$ condition $(2167.3 \mathrm{~cm})$. RL ranged from $8 \mathrm{~cm}$ to $74 \mathrm{~cm}$ in $\mathrm{P}$ stressed condition, while in case of $\mathrm{P}$ supplemented condition, it was raged from $7 \mathrm{~cm}$ to $92 \mathrm{~cm}$. In $\mathrm{P}$, tolerant genotypes root initiation and emergence is stimulated in P stress conditions (Den Herder et al., 2010; Postma et al., 2014). Since, deeper roots offer plants with improved access to stored water and nutrient uptake in the deeper layers of the soil substratum (Vejchasarn et al., 2016).

In controlled condition, some genotypes (ARB6, Shenong, Vandana, Azucena, IR84887 B-15, Samleshwari, DXDD-124-31, Swarna sub1, Bamleshwari, ARB 8, IR64) having very high RL but they fail to performed in $\mathrm{P}$ stress and having greatest reduction in their RL (Table 2). Effects of $P$ deficiency on the RL of 12 genotypes (Buddha, Ramjiyawan, MTU-1010, Pinkaeo, Desi Lal Dhan, R-RF-78, IR55419, Kalia, 
Annada, Abhaya, Cross 116 and SLO 16) were negligible and having no significant changes in their RL in contrasting $\mathrm{P}$ conditions. While the remaining 34 genotypes were considered as susceptible which having the significantly reduced RL in P deficiency (Figure 3A). Analysis of variations shows there is significant difference only for the phosphorus treatment (Table 3).

\section{Total Root Length (TRL)}

Total root length is the addition of length of the entire roots which includes the main root length and length of lateral root with their branching. Analysis of variations (Table 4) showed significant differences among varieties, phosphorus treatment and interaction between varieties and $P$ treatments. Overall TRL were decreased in P deficient condition compared to $\mathrm{P}$ sufficient one (Table 2 and Figure 3B), because root growth is inhibited during $\mathrm{P}$ stress and proportion of lateral root length was reduced with low $\mathrm{P}$ availability, depending on the genotype (Chiangmai et al., 2011; LopezBucio et al., 2011). In $\mathrm{P}$ stressed condition TRL ranged from $61.74 \mathrm{~cm}$ to $5560.9 \mathrm{~cm}$, while in case of $\mathrm{P}$ supplemented condition, it was rages from $28.09 \mathrm{~cm}$ to $5647.1 \mathrm{~cm}$. TRL having the lateral roots are considered the most active portion of the root system (Tuberosa 2012; Vejchasarn et al., 2016). It plays important role than the just RL in water and nutrient uptake and represent the majority of the root length of root systems (Rewald et al., 2011).

Out of 46 genotypes, 3 genotypes namely Buddha, RR-F-78 and Cross 116 (Figure 3B) are having the significantly enhanced TRL in phosphorus deficient condition and considered as tolerant genotypes since TRL as trait for evaluation. 10 genotypes (R-RF-69, DXDD-124-35, MTU-1010, Desi No 17, IR84978-B-60-4-1, Ramjiyawan, Annada, Bamleshwari, IR55419 and Kranti) were having the negligible change in their TRL (Figure 3B). While the remaining 33 genotypes having the significantly reduced TRL in $\mathrm{P}$ stressed condition compared to $\mathrm{P}$ sufficient one and since considered as the susceptible for the $\mathrm{P}$ deficiency. Highest increase in TRL was observed in Buddha $(+1833.64)$ whereas, the highest reduction was found in ARB6 (-5348.12).
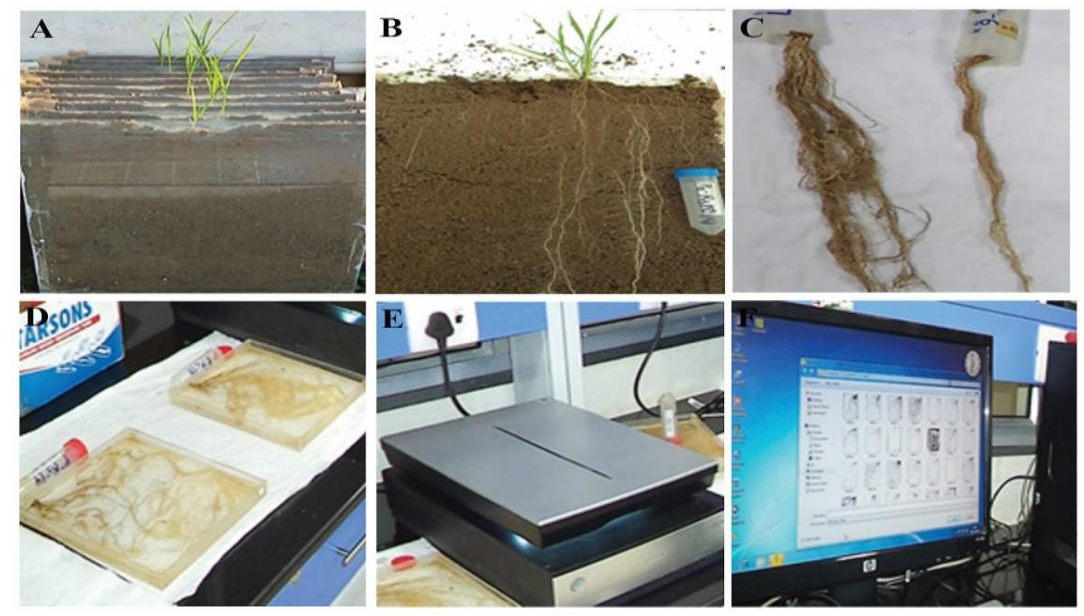

Fig.1 Work flow of the root morphology study using root rhizotron and WinRhizo image analysis system. A: Root rhizotron after 60 DAS; B: Opened rhizotron plate having the intact root and shoot; C: Separated cleaned root from shoot; D: Plastic acrylic container having separated root in water; E: Scanning in root scanner (Epson Perfection V700 Photo) for root image analysis; F: Scanned image and file of the resulted root 

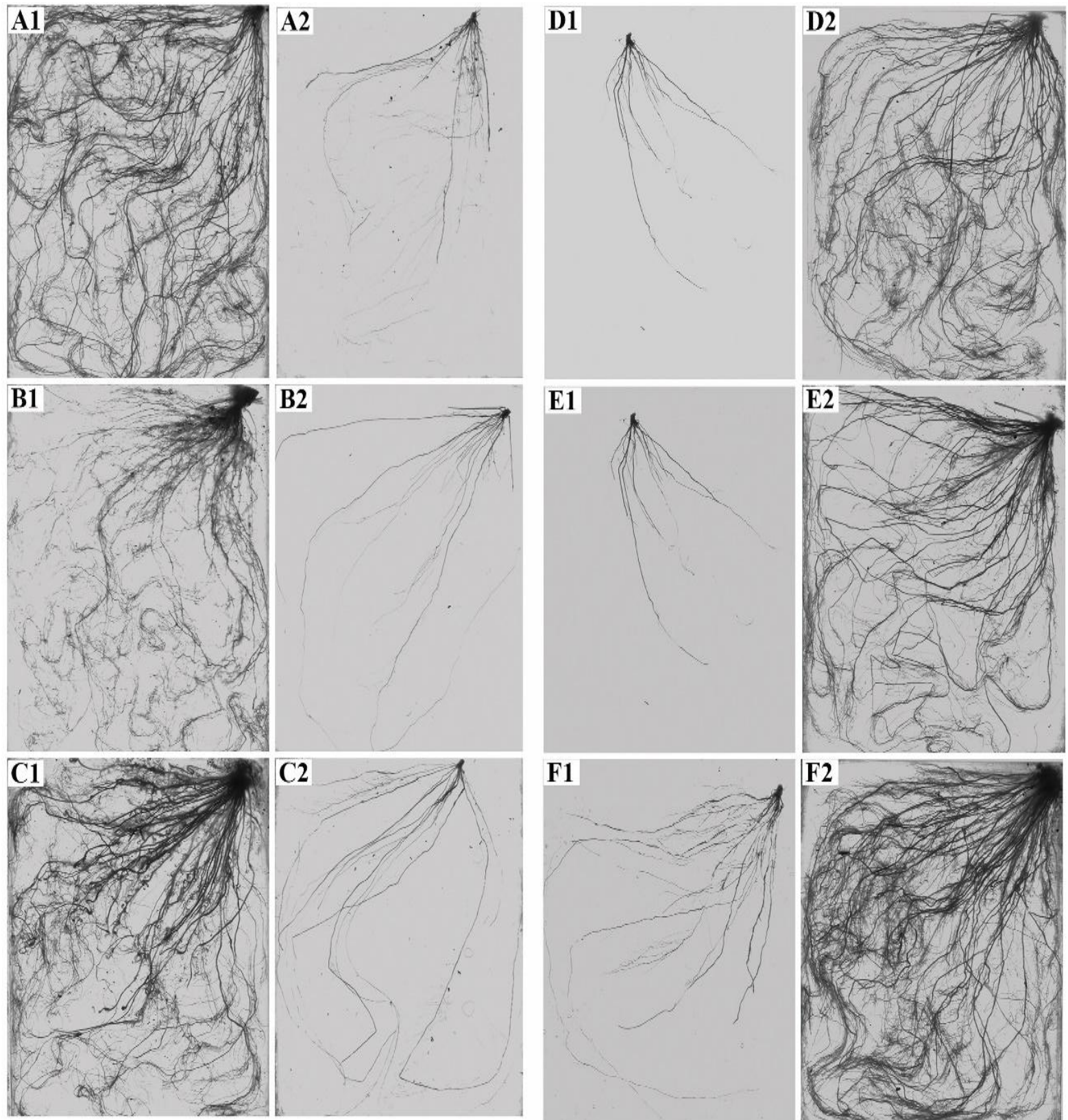

Fig.2 Root morphology of highly tolerant (A, B and C) and highly susceptible (D, E and F) rice genotypes under deficient and abundant phosphorus (P) supply. A1 and A2: Buddha under deficient and sufficient P, similarly; B1 and B2: R-RF-78 under deficient and sufficient P; C1 and C2: Cross 116 under deficient and sufficient P; D1 and D2: Vandana under deficient and sufficient P; E1 and E2: ARB6 under deficient and sufficient P;

F1 and F2: Samleshwari under deficient and sufficient $P$ 

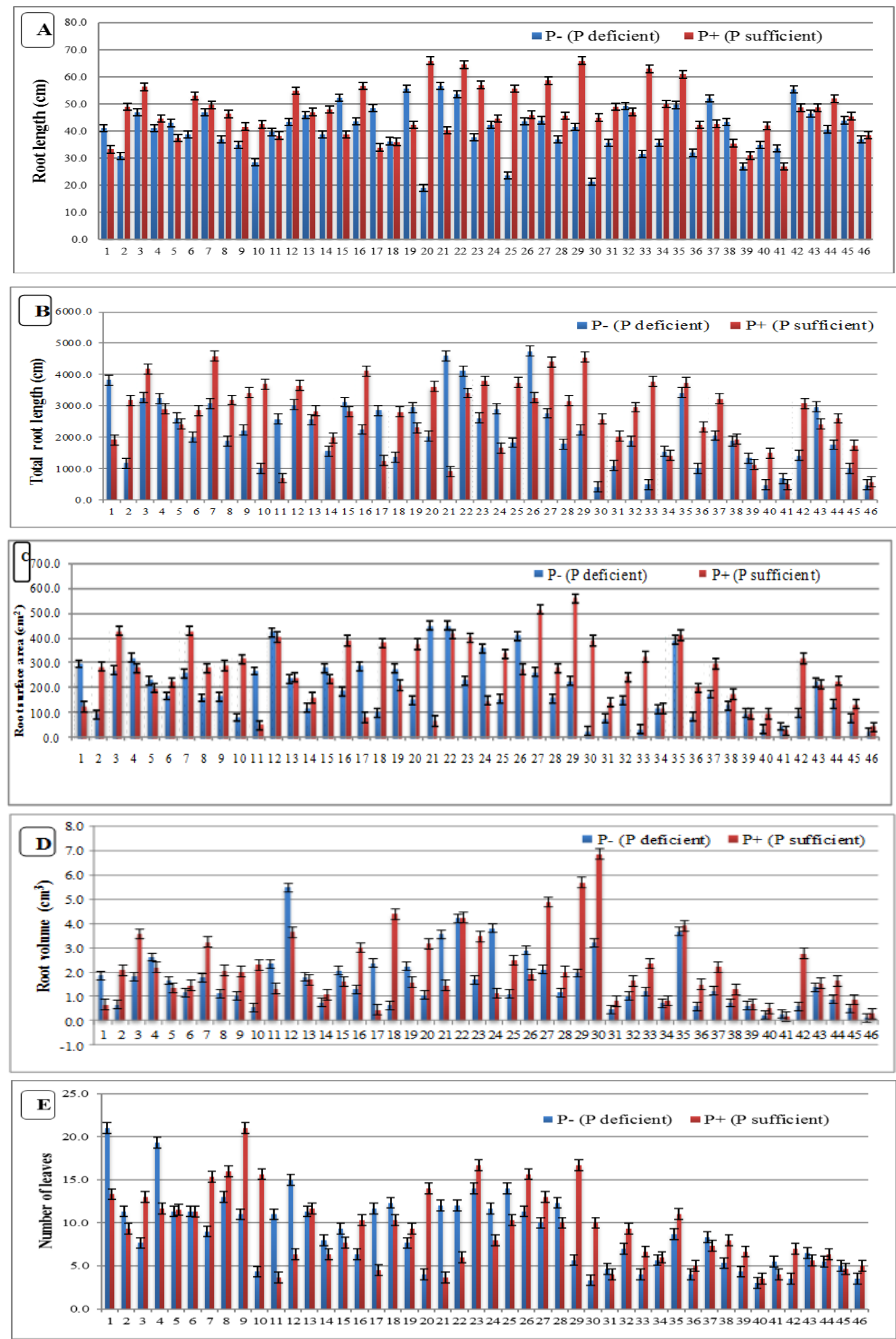

Fig.3 A: Root Length, B: Total Root Length, C: Total Surface Area, D: Total Surface Volume and $\mathbf{E}$ : Number of Leaves of 46 diverse rice genotypes under abundant and deficient phosphorus (P) supply. Error bars indicate the standard error of the means $(n=3)$ 
Table.1 Rice genotypes used for evaluation of phosphorous deficiency tolerance

\begin{tabular}{|c|c|}
\hline Name of the Genotype & Category/Type \\
\hline R-RF-78 & Advanced Breeding line- Rainfed conditions \\
\hline IR84887 B-15 & Advanced Breeding line- Rainfed conditions \\
\hline Sahbhagi dhan & Elite Variety \\
\hline IR84978-B-60-4-1 & Advanced Breeding line \\
\hline Annada & Variety \\
\hline IR64 & Variety \\
\hline Mahamaya & Variety \\
\hline Danteshwari & Variety \\
\hline Swarna & Variety \\
\hline Swarna-sub1 & Variety-Submergence tolerance \\
\hline Cross 116 & Germplasm \\
\hline Dagaddeshi & Landrace \\
\hline IR 36 & Elite Variety \\
\hline Laloo14 & Landrace \\
\hline Ramjiyawan & Landrace \\
\hline Kalokuchi & Landrace \\
\hline Pinkaeo & Landrace \\
\hline PM6004 & Variety \\
\hline MTU-1010 & Elite Variety \\
\hline ARB6 & Advanced Breeding line- Aerobic rice \\
\hline Buddha & Landrace \\
\hline Desi no 17 & Landrace \\
\hline DXDD-124-31 & Advanced Breeding line- Drought stress \\
\hline DXDD-124-35 & Advanced Breeding line- Drought stress \\
\hline Shenong & Landrace \\
\hline R-RF-69 & Advanced Breeding line- Drought stress \\
\hline R-RF-75 & Advanced Breeding line- Drought stress \\
\hline Purnima & Variety \\
\hline Samleshwari & Variety \\
\hline Vandana & Variety \\
\hline ARB 8 & Advanced Breeding line- Drought stress \\
\hline Abhaya & Variety \\
\hline Azucena & Landrace \\
\hline Bamleshwari & Variety \\
\hline Bakal & Landrace \\
\hline СТ9993 & Variety- Drought stress \\
\hline Desi Lal Dhan & Landrace \\
\hline IR55419 & Advanced Breeding line- Drought stress \\
\hline Kranti & Variety \\
\hline IBD1 & Variety- Drought stress \\
\hline SLO 16 & Landrace \\
\hline Kalia & Landrace \\
\hline Pratao & Landrace \\
\hline Chau dau & Landrace \\
\hline CR5272 & Landrace \\
\hline Dj-golon & Landrace \\
\hline
\end{tabular}


Table.2 Mean performance of root and shoot traits of 46 diverse rice genotypes in $\mathrm{P}$ sufficient and deficient condition

\begin{tabular}{|c|c|c|c|c|c|c|c|c|c|c|}
\hline \multirow{3}{*}{ Genotypes } & \multicolumn{8}{|c|}{ Root Traits } & \multirow{2}{*}{\multicolumn{2}{|c|}{$\frac{\text { Shoot Traits }}{\text { NoL }}$}} \\
\hline & \multicolumn{2}{|c|}{ RL } & \multicolumn{2}{|c|}{ TRL } & \multicolumn{2}{|c|}{ RSA } & \multicolumn{2}{|c|}{ RV } & & \\
\hline & $\mathrm{P}+$ & P- & $\mathrm{P}+$ & P- & $\mathrm{P}+$ & P- & $\mathrm{P}+$ & P- & $\mathrm{P}+$ & P- \\
\hline R-RF-78 & $33.3^{\mathrm{c}}$ & $42.0^{\mathrm{b}}$ & $387.9^{c}$ & $3823.3^{\mathrm{a}}$ & $28.2^{\mathrm{c}}$ & $274.2^{\mathrm{a}}$ & $0.16^{\mathrm{c}}$ & $1.92^{\mathrm{b}}$ & $3.3^{\mathrm{c}}$ & $21.0^{\mathrm{a}}$ \\
\hline IR84887 B-15 & $49.0^{b}$ & $30.8^{\mathrm{c}}$ & $3179.1^{\mathrm{a}}$ & $1166.2^{c}$ & $288.2^{b}$ & $97.3^{c}$ & $2.08^{\mathrm{b}}$ & $0.66^{\mathrm{c}}$ & $9.3^{c}$ & $11.3^{b}$ \\
\hline Sahbhagi dhan & $56.3^{b}$ & $47.0^{c}$ & $4179.9^{b}$ & $3254.4^{\mathrm{c}}$ & $432.3^{b}$ & $263.0^{\mathrm{c}}$ & $3.56^{\mathrm{b}}$ & $1.77^{\mathrm{c}}$ & $13.0^{\mathrm{a}}$ & $7.7^{\mathrm{c}}$ \\
\hline IR84978-B-60-4-1 & $44.7^{b}$ & $41.0^{c}$ & $2898.8^{\mathrm{c}}$ & $3233.3^{b}$ & $282.1^{\mathrm{c}}$ & $324.4^{b}$ & $2.19^{\mathrm{c}}$ & $2.61^{b}$ & $11.7^{\mathrm{c}}$ & $19.3^{\mathrm{a}}$ \\
\hline Annada & $36.7^{\mathrm{c}}$ & $43.0^{b}$ & $2376.1^{\mathrm{c}}$ & $2614.6^{b}$ & $201.0^{\mathrm{c}}$ & $232.1^{\mathrm{b}}$ & $1.25^{\mathrm{c}}$ & $1.65^{b}$ & $11.7^{b}$ & 11.3 \\
\hline IR64 & $53.0^{\mathrm{b}}$ & $38.7^{\mathrm{c}}$ & $2844.9^{\mathrm{b}}$ & $1997.4^{\mathrm{c}}$ & $226.1^{\mathrm{b}}$ & $170.0^{\mathrm{c}}$ & $1.44^{\mathrm{b}}$ & $1.17^{\mathrm{c}}$ & $11.3^{\mathrm{b}}$ & $11.3^{\mathrm{b}}$ \\
\hline Mahamaya & $49.7^{\mathrm{b}}$ & $47.0^{c}$ & $4591.4^{\mathrm{b}}$ & $3077.8^{\mathrm{c}}$ & $431.0^{b}$ & $261.5^{\mathrm{c}}$ & $3.23^{b}$ & $1.77^{\mathrm{c}}$ & $15.3^{\mathrm{a}}$ & $9.0^{c}$ \\
\hline Danteshwari & $46.3^{b}$ & $37.0^{c}$ & $3183.5^{b}$ & $1885.6^{c}$ & $282.8^{b}$ & $162.5^{\mathrm{c}}$ & $2.06^{b}$ & $1.12^{\mathrm{c}}$ & 16.0 & $13.0^{\mathrm{c}}$ \\
\hline Swarna & $41.7^{\mathrm{b}}$ & $34.8^{\mathrm{c}}$ & $3420.8^{\mathrm{b}}$ & $2216.3^{c}$ & $293.6^{b}$ & $169.2^{\mathrm{c}}$ & $2.02^{b}$ & $1.04^{\mathrm{c}}$ & $21.0^{\mathrm{a}}$ & $11.0^{\mathrm{c}}$ \\
\hline Swarna-sub1 & $42.5^{\mathrm{b}}$ & $28.5^{\mathrm{c}}$ & $3692.3^{\mathrm{a}}$ & $1009.9^{c}$ & $318.3^{\mathrm{a}}$ & $84.0^{\mathrm{c}}$ & $2.29^{b}$ & $0.56^{\mathrm{c}}$ & $15.7^{\mathrm{a}}$ & $4.3^{c}$ \\
\hline Cross 116 & $38.3^{\mathrm{c}}$ & $39.7^{b}$ & $671.3^{c}$ & $2578.2^{a}$ & $52.6^{\mathrm{c}}$ & $270.8^{\mathrm{a}}$ & $0.33^{\mathrm{c}}$ & $2.34^{\mathrm{b}}$ & $3.7^{\mathrm{c}}$ & $11.0^{\mathrm{a}}$ \\
\hline Dagaddeshi & $54.9^{b}$ & $43.3^{\mathrm{c}}$ & $3643.9^{b}$ & $3038.0^{\mathrm{b}}$ & $407.8^{\mathrm{c}}$ & $426.6^{b}$ & $3.64^{\mathrm{c}}$ & $5.48^{b}$ & $6.3^{\mathrm{c}}$ & $15.0^{\mathrm{a}}$ \\
\hline IR 36 & $47.0^{b}$ & $46.0^{c}$ & $2843.5^{b}$ & $2566.4^{\mathrm{c}}$ & $244.8^{b}$ & $238.6^{c}$ & $1.68^{\mathrm{c}}$ & $1.80^{\mathrm{b}}$ & 11.7 & $11.3^{\mathrm{c}}$ \\
\hline Laloo14 & $48.0^{b}$ & $38.7^{\mathrm{c}}$ & $1980.9^{b}$ & $1556.2^{\mathrm{c}}$ & $162.8^{\mathrm{b}}$ & $123.1^{\mathrm{c}}$ & $1.07^{\mathrm{b}}$ & $0.74^{\mathrm{c}}$ & $6.3^{c}$ & $8.0^{\mathrm{b}}$ \\
\hline Ramjiyawan & $38.8^{\mathrm{c}}$ & $52.3^{b}$ & $2808.4^{\mathrm{c}}$ & $3120.2^{b}$ & $239.4^{\mathrm{c}}$ & $282.2^{b}$ & $1.59^{\mathrm{c}}$ & $2.06^{b}$ & $7.7^{c}$ & $9.3^{b}$ \\
\hline Kalokuchi & $56.7^{b}$ & $43.7^{\mathrm{c}}$ & $4111.3^{b}$ & $2244.6^{c}$ & $393.2^{b}$ & $190.2^{\mathrm{c}}$ & $3.00^{\mathrm{b}}$ & $1.29^{\mathrm{c}}$ & $10.3^{\mathrm{b}}$ & $6.3^{\mathrm{c}}$ \\
\hline Pinkaeo & $35.3^{\mathrm{c}}$ & $48.5^{b}$ & $4873.0^{\mathrm{a}}$ & $2857.4^{b}$ & $81.5^{\mathrm{c}}$ & $290.1^{\mathrm{b}}$ & $0.37^{\mathrm{c}}$ & $2.35^{b}$ & $4.7^{c}$ & $11.7^{\mathrm{a}}$ \\
\hline PM6004 & $36.0^{c}$ & $36.3^{c}$ & $2803.2^{b}$ & $1324.7^{\mathrm{c}}$ & $384.2^{\mathrm{a}}$ & $99.9^{\mathrm{c}}$ & $4.39^{\mathrm{a}}$ & $0.64^{\mathrm{c}}$ & $10.3^{\mathrm{c}}$ & $12.3^{\mathrm{b}}$ \\
\hline MTU-1010 & $42.3^{c}$ & $55.7^{b}$ & $2297.4^{c}$ & $2943.1^{b}$ & $212.4^{\mathrm{c}}$ & $281.0^{b}$ & $1.57^{\mathrm{c}}$ & $2.23^{b}$ & $9.3^{b}$ & $7.7^{c}$ \\
\hline ARB6 & $61.7^{\mathrm{a}}$ & $19.0^{\mathrm{c}}$ & $3707.0^{\mathrm{a}}$ & $225.9^{c}$ & $361.1^{\mathrm{a}}$ & $12.9^{\mathrm{c}}$ & $3.25^{\mathrm{a}}$ & $0.06^{\mathrm{c}}$ & $15.0^{\mathrm{a}}$ & $4.0^{\mathrm{c}}$ \\
\hline Buddha & $40.3^{c}$ & $56.7^{\mathrm{b}}$ & $902.6^{c}$ & $4603.2^{\mathrm{a}}$ & $70.1^{\mathrm{c}}$ & $451.4^{\mathrm{a}}$ & $0.44^{\mathrm{c}}$ & $3.55^{\mathrm{a}}$ & $3.7^{b}$ & $12.0^{\mathrm{a}}$ \\
\hline Desi no 17 & $61.7^{b}$ & $53.7^{\mathrm{c}}$ & $3679.3^{c}$ & $4108.2^{b}$ & $387.7^{\mathrm{c}}$ & $453.5^{b}$ & $3.29^{\mathrm{c}}$ & $4.21^{b}$ & $6.3^{\mathrm{b}}$ & $12.0^{\mathrm{a}}$ \\
\hline DXDD-124-31 & $57.0^{\mathrm{b}}$ & $37.7^{\mathrm{c}}$ & $3800.4^{b}$ & $2609.0^{c}$ & $405.9^{b}$ & $233.9^{c}$ & $3.47^{\mathrm{b}}$ & $1.68^{\mathrm{c}}$ & $16.7^{\mathrm{c}}$ & $14.0^{\mathrm{c}}$ \\
\hline DXDD-124-35 & $44.7^{b}$ & $42.3^{c}$ & $1648.2^{\mathrm{c}}$ & $2902.8^{\mathrm{b}}$ & $151.6^{\mathrm{c}}$ & $360.8^{\mathrm{b}}$ & $1.13^{\mathrm{c}}$ & $3.80^{\mathrm{b}}$ & $8.0^{c}$ & $11.7^{b}$ \\
\hline Shenong & $55.7^{\mathrm{a}}$ & $23.7^{\mathrm{c}}$ & $3739.3^{\mathrm{a}}$ & $1826.7^{\mathrm{c}}$ & $340.1^{b}$ & $158.8^{\mathrm{c}}$ & $2.48^{\mathrm{b}}$ & $1.10^{\mathrm{c}}$ & $10.3^{\mathrm{b}}$ & $14.0^{\mathrm{b}}$ \\
\hline R-RF-69 & $46.0^{\mathrm{b}}$ & $43.7^{\mathrm{c}}$ & $3264.8^{\mathrm{c}}$ & $4751.9^{b}$ & $278.5^{\mathrm{c}}$ & $413.0^{\mathrm{b}}$ & $1.90^{\mathrm{b}}$ & $2.89^{\mathrm{b}}$ & $15.7^{\mathrm{b}}$ & $11.3^{\mathrm{c}}$ \\
\hline R-RF-75 & $58.7^{\mathrm{b}}$ & $44.0^{c}$ & $4224.1^{\mathrm{b}}$ & $2764.2^{\mathrm{c}}$ & $512.2^{\mathrm{a}}$ & $266.1^{c}$ & $4.67^{b}$ & $2.11^{\mathrm{c}}$ & $13.0^{\mathrm{b}}$ & $10.0^{\mathrm{c}}$ \\
\hline Purnima & $45.7^{b}$ & $37.0^{\mathrm{c}}$ & $3161.2^{b}$ & $1776.4^{\mathrm{c}}$ & $282.6^{\mathrm{b}}$ & $159.8^{\mathrm{c}}$ & $2.02^{b}$ & $1.16^{\mathrm{c}}$ & $10.0^{\mathrm{c}}$ & $12.3^{b}$ \\
\hline Samleshwari & $66.0^{\mathrm{a}}$ & $41.7^{\mathrm{c}}$ & $4557.5^{\mathrm{a}}$ & $2225.7^{\mathrm{c}}$ & $563.7^{\mathrm{a}}$ & $231.3^{\mathrm{c}}$ & $5.67^{\mathrm{a}}$ & $1.96^{\mathrm{c}}$ & $16.7^{\mathrm{a}}$ & $5.7^{\mathrm{c}}$ \\
\hline Vandana & $45.0^{\mathrm{a}}$ & $21.3^{\mathrm{c}}$ & $2578.2^{\mathrm{a}}$ & $410.7^{\mathrm{c}}$ & $392.4^{\mathrm{a}}$ & $28.8^{\mathrm{c}}$ & $5.65^{\mathrm{a}}$ & $0.29^{\mathrm{c}}$ & $10.0^{\mathrm{a}}$ & $3.3^{\mathrm{c}}$ \\
\hline ARB 8 & $50.3^{b}$ & $35.7^{\mathrm{c}}$ & $2014.8^{\mathrm{b}}$ & $1092.0^{\mathrm{c}}$ & $142.9^{\mathrm{b}}$ & $79.9^{\mathrm{c}}$ & $0.79^{b}$ & $0.47^{\mathrm{c}}$ & $4.3^{c}$ & $4.7^{\mathrm{b}}$ \\
\hline Abhaya & $47.0^{c}$ & $49.3^{b}$ & $3384.5^{b}$ & $1769.5^{\mathrm{c}}$ & $249.9^{b}$ & $157.6^{\mathrm{c}}$ & $1.33^{\mathrm{b}}$ & $0.72^{\mathrm{c}}$ & $9.3^{\mathrm{b}}$ & $7.0^{\mathrm{c}}$ \\
\hline Azucena & $63.0^{\mathrm{a}}$ & $31.7^{\mathrm{c}}$ & $3777.3^{\mathrm{a}}$ & $605.4^{\mathrm{c}}$ & $329.6^{\mathrm{a}}$ & $44.5^{\mathrm{c}}$ & $2.34^{\mathrm{b}}$ & $0.20^{\mathrm{c}}$ & $6.7^{b}$ & $4.0^{\mathrm{c}}$ \\
\hline Bamleshwari & $51.7^{\mathrm{b}}$ & $35.7^{\mathrm{c}}$ & $1413.6^{\mathrm{c}}$ & $1537.6^{\mathrm{c}}$ & $120.4^{b}$ & $118.6^{\mathrm{c}}$ & $0.83^{b}$ & $0.74^{\mathrm{c}}$ & $6.0^{b}$ & $5.7^{\mathrm{c}}$ \\
\hline Bakal & $60.7^{b}$ & $49.7^{\mathrm{c}}$ & $3675.4^{b}$ & $3364.0^{b}$ & $377.0^{\mathrm{c}}$ & $381.8^{\mathrm{b}}$ & $3.72^{b}$ & $3.43^{\mathrm{c}}$ & $11.3^{\mathrm{b}}$ & $8.7^{\mathrm{c}}$ \\
\hline CT9993 & $42.3^{b}$ & $32.0^{\mathrm{c}}$ & $2319.6^{\mathrm{b}}$ & $1002.4^{\mathrm{c}}$ & $203.5^{b}$ & $85.1^{\mathrm{c}}$ & $1.49^{\mathrm{b}}$ & $0.60^{\mathrm{c}}$ & $5.0^{\mathrm{b}}$ & $4.0^{\mathrm{c}}$ \\
\hline Desi Lal Dhan & $42.7^{c}$ & $52.0^{\mathrm{b}}$ & $3335.3^{b}$ & $2052.1^{\mathrm{c}}$ & $282.4^{\mathrm{b}}$ & $178.0^{\mathrm{c}}$ & $2.45^{\mathrm{b}}$ & $1.23^{\mathrm{c}}$ & $7.3^{\mathrm{c}}$ & $8.3^{\mathrm{b}}$ \\
\hline IR55419 & $35.7^{\mathrm{c}}$ & $43.3^{b}$ & $1770.1^{\mathrm{c}}$ & $1879.9^{\mathrm{c}}$ & $177.7^{\mathrm{b}}$ & $131.9^{\mathrm{c}}$ & $1.08^{\mathrm{b}}$ & $0.74^{\mathrm{c}}$ & $8.0^{\mathrm{b}}$ & $5.3^{\mathrm{c}}$ \\
\hline Kranti & $31.0^{\mathrm{b}}$ & $27.0^{c}$ & $1233.6^{\mathrm{c}}$ & $1326.7^{\mathrm{c}}$ & $116.9^{b}$ & $101.2^{\mathrm{c}}$ & $0.69^{\mathrm{b}}$ & $0.61^{\mathrm{c}}$ & $6.7^{b}$ & $4.3^{\mathrm{c}}$ \\
\hline IBD1 & $44.0^{b}$ & $35.0^{\mathrm{c}}$ & $1502.0^{b}$ & $477.7^{\mathrm{c}}$ & $92.7^{b}$ & $36.0^{\mathrm{c}}$ & $0.47^{b}$ & $0.22^{\mathrm{c}}$ & $2.3^{\mathrm{c}}$ & $3.0^{\mathrm{b}}$ \\
\hline SLO 16 & $32.7^{\mathrm{c}}$ & $33.7^{b}$ & $834.8^{b}$ & $679.6^{c}$ & $51.2^{b}$ & $47.9^{c}$ & $0.27^{\mathrm{c}}$ & $0.27^{b}$ & $4.0^{c}$ & $5.7^{b}$ \\
\hline Kalia & $48.7^{c}$ & $55.3^{b}$ & $3076.5^{b}$ & $1407.5^{c}$ & $320.1^{\mathrm{a}}$ & $101.6^{\mathrm{c}}$ & $2.75^{\mathrm{b}}$ & $0.60^{\mathrm{c}}$ & $7.0^{\mathrm{b}}$ & $3.7^{\mathrm{c}}$ \\
\hline Pratao & $48.7^{b}$ & $46.0^{c}$ & $2411.5^{b}$ & $1974.8^{b}$ & $215.5^{\mathrm{c}}$ & $248.9^{b}$ & $1.54^{\mathrm{c}}$ & $1.64^{b}$ & $5.7^{b}$ & $4.3^{\mathrm{c}}$ \\
\hline Chau dau & $52.0^{\mathrm{b}}$ & $40.7^{\mathrm{c}}$ & $2600.4^{b}$ & $1761.8^{\mathrm{c}}$ & $229.5^{b}$ & $139.4^{\mathrm{c}}$ & $1.64^{\mathrm{b}}$ & $0.88^{\mathrm{c}}$ & $6.3^{\mathrm{b}}$ & $5.3^{\mathrm{c}}$ \\
\hline CR5272 & $45.5^{\mathrm{b}}$ & $44.0^{c}$ & $1743.9^{b}$ & $1007.9^{c}$ & $137.7^{b}$ & $80.2^{c}$ & $0.87^{\mathrm{b}}$ & $0.51^{\mathrm{c}}$ & $4.7^{c}$ & $5.3^{\mathrm{b}}$ \\
\hline Dj-golon & $38.7^{\mathrm{b}}$ & $37.0^{c}$ & $2593.1^{\text {a }}$ & $469.20^{c}$ & $44.53^{b}$ & $25.4^{\mathrm{c}}$ & $0.26^{b}$ & $0.11^{\mathrm{c}}$ & $5.0^{6}$ & $3.7^{\mathrm{c}}$ \\
\hline Grand Mean & 2167.3 & 1862.7 & 129737.0 & 91120.8 & 11800.3 & 8969.0 & 94.4 & 69.0 & 432.7 & 405.3 \\
\hline
\end{tabular}

RL: Root length, TRL: Total root length, RSA: Root surface area, RV: Root volume, NoL: Number of leaves, (P+): $\mathrm{P}$ sufficient, (P-): $\mathrm{P}$ deficient. Means denoted by the same letters within each column are not significantly different at $\mathrm{P}<0.05$. 
Table.3 The effects of genotype and phosphorus treatment on mean sum of square of root traits and number of leaves

\begin{tabular}{|l|l|l|l|}
\hline \multirow{2}{*}{\multicolumn{1}{c|}{ Trait }} & \multicolumn{3}{c|}{ Mean Sum of Square (MSS) } \\
\cline { 2 - 4 } & \multicolumn{1}{|c|}{ Variety (V) } & \multicolumn{1}{c|}{ Phosphorus (P) } & \multicolumn{1}{c|}{ Interaction (VxP) } \\
\hline Root Length (RL) & 225.97 & 3026.80 & 237.19 \\
\hline Total Root Length (TRL) & 4034574.00 & 34689630 & 3249525.00 \\
\hline Root Surface Area (RSA) & 54068.42 & 261409.80 & 39705.94 \\
\hline Root Volume (RV) & 5.78 & 21.10 & 4.24 \\
\hline Number of leaves (NoL) & 65.78 & 10.96 & 46.30 \\
\hline
\end{tabular}

Table.4 Scoring scheme for phosphorus deficiency tolerance (PDT) screening of rice genotypes

\begin{tabular}{|c|c|}
\hline Type & Variety \\
\hline $\begin{array}{l}\text { Tolerant } \\
\text { Moderately } \\
\text { tolerant/ Not } \\
\text { affected by P } \\
\text { treatment }\end{array}$ & $\begin{array}{l}\text { Buddha, RR-F-78, Cross } 116 \\
\text { DXDD-124-35, Pinkaeo, R-RF-69, MTU-1010, Desi } \\
\text { Ramjiyawan, IR84978-B-60-4-1, Pratao, Annada, Dagaddeshi }\end{array}$ \\
\hline P Sensitive & $\begin{array}{l}\text { Vandana, ARB6, Samleshwari, Azucena, PM6004, R-RF-75, Swarna } \\
\text { sub1, Kalia, Kalokuchi, IR84887 B-15, Shenong, DXDD-124-31, } \\
\text { Mahamaya, Sahbhagi Dhan, Swarna, Purnima, Danteshwari, CT9993, } \\
\text { Desi Lal Dhan, Abhaya, Chau Dau, ARB 8, CR5272, IBD1, IR64, } \\
\text { IR55419, Laloo14, DJ Golon, Kranti, IR 36, SLO 16, Bamleshwari, } \\
\text { Bakal. }\end{array}$ \\
\hline
\end{tabular}

Rice genotypes listed in the order of decreasing PDT. The classification was based on the five principle components calculated using Root length, Total root length, Root surface area, Root volume and Number of leaves in contrasting $\mathrm{P}$ conditions.

\section{Root Surface Area (RSA) and Root Volume (RV)}

Overall analysis across the $\mathrm{P}$ treatment showed that RSA decreased in case of $\mathrm{P}$ deficient condition compared to $\mathrm{P}$ sufficient. In $\mathrm{P}$ stressed condition RSA ranges from $5.97 \mathrm{~cm}^{2}$ to $923.2 \mathrm{~cm}^{2}$, while in case of $\mathrm{P}$ supplemented condition, it was rages from $2.15 \mathrm{~cm}^{2}$ to $918.90 \mathrm{~cm}^{2}$. Analysis across the $P$ levels showed that 3 genotypes namely Buddha, RR-F-78 and Cross 116 (Figure 3C) were having significantly increased RSA in $\mathrm{P}$ deficient condition and considered as tolerant for P stress. 11 genotypes (DXDD-124-35, Pinkaeo, R-RF-69, MTU1010, Desi No 17, Ramjiyawan, IR84978-B-60-4-1, Pratao,
Annada, Dagaddeshi and Bakal) were having negligible effect on RSA on $\mathrm{P}$ treatment. While the remaining 32 genotypes having the significantly reduced RSA and since considered as the susceptible for the P stress for RSA. Highest increase in RSA was observed in Buddha (+163.96) whereas, the highest reduction was found in Vandana (580.96). Enhanced root traits like length and surface area could have contributed to the high uptake of $\mathrm{P}$ (Wang et al., 2016).

Across the P treatments RV was decreased in $\mathrm{P}$ deficient condition as compare to the $\mathrm{P}$ sufficient one. In $\mathrm{P}$ stressed condition RV ranged from $0.028 .97 \mathrm{~cm}^{3}$ to $-14.08 \mathrm{~cm}^{3}$, while in case of $\mathrm{P}$ supplemented condition, it was 
raged from $0.013 \mathrm{~cm}^{3}$ to $14.03 \mathrm{~cm}^{3}$. Analysis across the $\mathrm{P}$ levels showed that Buddha (Figure 3D) were having significantly increased $\mathrm{RV}$ in $\mathrm{P}$ deficient condition and considered as tolerant for $\mathrm{P}$ stress. 14 genotypes (DXDD-124-35, Cross 116, Pinkaeo, Dagaddeshi, RR-F-78, R-RF-69, DESI NO 17, MTU1010, Ramjiyawan, IR84978-B-60-4-1, Annada, IR36, Pratao, and SLO 16) were having the negligible or non-significant effect on $\mathrm{P}$ treatment and since negligible change in $\mathrm{RV}$ among the $\mathrm{P}$ treatments. While the remaining 31 genotypes having the significantly reduced $\mathrm{RV}$ and considered as the susceptible for the P stress. Highest increase in RV was observed in Buddha (+0.42) whereas, the highest reduction was found in Vandana (-8.04).

$\mathrm{P}$ fertilization increases root biomass, total $\mathrm{P}$ in the plant tissues and $\mathrm{P}$ uptake. Our results show good agreement with previous studies of Teng et al., 2013 and Wang et al., 2016. The formation of root branching is a significant determinant of overall RSA and RV (Figure 2). $P$ deficiency conditions stimulate the proliferation of root hairs and lateral roots, and these traits can contribute $70 \%$ or more of the total root surface area and can be responsible for up to $90 \%$ of $\mathrm{P}$ acquired (Bates et al., 2001; Haling et al., 2013).

Lateral roots add to the total root biomass, RSA and RV. Analysis of variance showed significant difference among all genotypes, $\mathrm{P}$ treatment and interaction among varieties and $P$ treatment (Table 2 and 3 ).

\section{Number of leaves (NoL)}

Overall analysis showed decrease in number of leaves in case of $\mathrm{P}$ stressed condition compared to the sufficient condition. In $\mathrm{P}$ stressed condition NoL ranges from 1 to 25 , while in case of $\mathrm{P}$ supplemented condition, it was rages from 2 to 31 . Out of 46 genotypes 7 genotypes (RR-F-78, Dagaddeshi, Buddha, IR84978-B-60-4-1, Cross 116, Pinkaeo and Desi No 17) were having significantly increased NoL in $\mathrm{P}$ deficient condition and considered as tolerant for P stress. Since, 13 genotypes (DXDD-124-35, Shenong, Purnima, IR84887 B-15, PM6004, Laloo14, Ramjiyawan, SLO 16, Desi Lal Dhan, IBD1, CR5272, ARB 8 and IR64) were having notsignificant change in NoL among the $\mathrm{P}$ treatment. While the remaining 26 genotypes having the significantly reduced NoL and since considered as the susceptible for the $\mathrm{P}$ deficiency.

Highest increase in NoL was observed in RRF-78 (+12.45) whereas, the highest reduction was found in Swarna sub1 (-16.55). Analysis of variations shows there is significant difference for genotypes and interaction among the varieties and $\mathrm{P}$ treatment and not for the phosphorus treatment (Table 3).

This study provided evidence that root trait in rice plays important role in $\mathrm{P}$ deficiency tolerance. Phosphorus deficiency was highly influenced by genotypic differences and treatment of P. Under P stressed condition there is a significant enhancement in all the root traits in tolerant rice genotypes (Figure 2). In our experiment, we found that Buddha, RR-F-78 and Cross 116 are highest phosphorous deficiency tolerant rice genotypes. Vandana, ARB6 and Samleshwari are having the good root growth in the $\mathrm{P}$ sufficient condition but having greatest reduction growth of root trait and considered as highly susceptible rice genotypes (Table 3 ).

High yielding genotypes like Swarna, Swarna sub1, Shenong are very popular over the country for their yield performance is found susceptible for P deficiency, likely genotypes Sahbhagi dhan known for $\mathrm{P}$ tolerant in previous studies, but in our study, we found as the P susceptible. Selected parents on the 
basis of root traits can be further used for future breeding programs and genetic studies on PDT to identify potentially novel genetic mechanisms.

\section{References}

Abel S, Ticconi C A and Delatorre C A.2002. Phosphate sensing in higher plants. Physiologia Plantarum 115: 1-8.

Aluwihare, Y., M. Ishan, M. Chamikara, C. Weebadde, D. Sirisena, W. Samarasinghe, and S. Sooriyapathirana. 2016. Characterization and selection of phosphorus deficiency tolerant rice genotypes in Sri Lanka. Rice Science 23:184-195.

Bates, T. R. and J. P. Lynch. 2001. Root hairs confer a competitive advantage under low phosphorus availability. Plant and Soil 236:243-250.

Batjes N.1997. A world dataset of derived soil properties by FAO-UNESCO soil unit for global modelling. Soil use and management 13: 9-16.

Chiangmai P N and Yodmingkhwan P.2011. Competition of root and shoot growth between cultivated rice (Oryza sativa L.) and common wild rice (Oryza rufipogon Griff.) grown under different phosphorus levels. Journal of Science and Technology 33: 685-692.

Chin, J. H., X. Lu, S. M. Haefele, R. Gamuyao, A. Ismail, M. Wissuwa, and S. Heuer. 2010. Development and application of gene-based markers for the major rice QTL Phosphorus uptake 1. Theoretical and Applied Genetics 120:1073-1086.

Cordell D, Drangert J, Whit S. 2009. The story of phosphorus: Global food security and food for thought. Global Environmental Change 19: 292-305.

Den Herder, G., G. Van Isterdael, T. Beeckman, and I. De Smet. 2010. The roots of a new green revolution. Trends in plant science 15:600-607.

Fitter A.2002. Characteristics and functions of root systems. Plant roots: the hidden half: 21-50.

Gupta A, Kakade D. P., Singh J., Janjal P.H., Verulkar S.B. and Banerjee S. 2016. Characterization of Rice Root Transcriptome under Phosphorus Deficiency Stress. International Journal Current Microbiology Applied Sciences 5:273-279.

Haling, R. E., L. K. Brown, A. G. Bengough, I. M. Young, P. D. Hallett, P. J. White, and T. S. George. 2013. Root hairs improve root penetration, root-soil contact, and phosphorus acquisition in soils of different strength. Journal of Experimental Botany 64:3711-3721.

Jian-chang Y.2011. Relationships of rice root morphology and physiology with the formation of grain yield and quality and the nutrient absorption and utilization. Scientia Agricultura Sinica 1: 006.

Jones, D. L. 1998. Organic acids in the rhizosphere-a critical review. Plant Soil 205:25-44.

Kirk $G$ and DU L.1997. Changes in rice root architecture, porosity, and oxygen and proton release under phosphorus deficiency. New Phytol., 135: 191-200.

Lim J H,Chung I-M, Ryu S S,Park M R and Yun S J.2003. Differential responses of rice acid phosphatase activities and isoforms to phosphorus deprivation. $J$. Biochem. Mol. Biol., 36: 597-602.

Lopez-Bucio, J., A. Cruz-Ramırez, and L. Herrera-Estrella. 2003. The role of nutrient availability in regulating root architecture. Current opinion in plant biology 6:280-287.

Lynch J P.2007. Turner review no. 14. Roots of the second green revolution. Australian Journal of Botany 55: 493-512.

Lynch J P.2011. Root phenes for enhanced soil exploration and phosphorus acquisition: tools for future crops. Plant physiology 156: 1041-1049.

Lynch J.1995. Root architecture and plant productivity. Plant physiology 109: 7.

Nielsen N E.1979. Plant factors determining the efficiency of nutrient uptake from soils. Acta Agriculturae Scandinavica 29: 81- 
84.

Paez-Garcia, A., C. M. Motes, W.-R. Scheible, R. Chen, E. B. Blancaflor, and M. J. Monteros. 2015. Root traits and phenotyping strategies for plant improvement. Plants 4:334-355.

Panigrahy M,Rao D N and Sarla N.2009. Molecular mechanisms in response to phosphate starvation in rice. Biotechnology advances 27: 389-397.

Poirier Y and Bucher M.2002. Phosphate transport and homeostasis in Arabidopsis. The Arabidopsis Book: e0024.

Postma, J. A., A. Dathe, and J. P. Lynch. 2014. The optimal lateral root branching density for maize depends on nitrogen and phosphorus availability. Plant Physiol 166:590-602.

Rewald, B., J. E. Ephrath, and S. Rachmilevitch. 2011. A root is a root is a root? Water uptake rates of Citrus root orders. Plant Cell Environ 34:33-42.

Richardson, A. E., P. J. Hocking, R. J. Simpson, and T. S. George. 2009. Plant mechanisms to optimise access to soil phosphorus. Crop and Pasture Science 60:124-143.

Rose, T. J. and M. Wissuwa. 2012. Rethinking internal phosphorus utilization efficiency: a new approach is needed to improve PUE in grain crops. Advances in agronomy 116:185-217.

Sarker B C and Karmoker J.2009. Effects of phosphorus deficiency on the root growth of lentil seedlings grown in rhizobox. Bangladesh Journal of Botany 38: $215-218$.
Teng, W., Y. Deng, X.-P. Chen, X.-F. Xu, R.Y. Chen, Y. Lv, Y.-Y. Zhao, X.-Q. Zhao, X. He, and B. Li. 2013. Characterization of root response to phosphorus supply from morphology to gene analysis in field-grown wheat. Journal of Experimental Botany 64:1403-1411.

Tuberosa, R. 2012. Phenotyping for drought tolerance of crops in the genomics era. Frontiers in Physiology 3: 347.

Vejchasarn, P., J. P. Lynch, and K. M. Brown. 2016. Genetic Variability in Phosphorus Responses of Rice Root Phenotypes. Rice 9:1-16.

Wang Y, Thorup-Kristensen K, Stoumann Jensen L and Magid J.2016. Vigorous root growth is a better indicator of early nutrient uptake than root hair traits in spring wheat grown under low fertility. Frontiers in Plant Science 7: 865.

Wissuwa M, Gamat G and Ismail A M.2005. Is root growth under phosphorus deficiency affected by source or sink limitations? Journal of Experimental Botany 56: 1943-1950.

Wissuwa M.2005. Combining a modelling with a genetic approach in establishing associations between genetic and physiological effects in relation to phosphorus uptake. Plant and Soil 269: 57-68.

Wu W and Cheng S.2014. Root genetic research, an opportunity and challenge to rice improvement. Field Crops Research 165: 111-124.

\section{How to cite this article:}

Datta P. Kakade, Jyoti Singh, Mayur R. Wallalwar, Arun Janjal, Anjali Gupta, Rishiraj Raghuvanshi, Miranda Kongbrailatpam, Satish B. Verulkar and Shubha Banerjee. 2017. Differential Response of Root Morphology of Rice (Oryza sativa L.) Genotypes under Different Phosphorus Conditions. Int.J.Curr.Microbiol.App.Sci. 6(7): 149-160.

doi: https://doi.org/10.20546/ijcmas.2017.607.018 